\title{
A SHORT PROOF OF THE FOURIER INVERSION FORMULA
}

\section{ALAIN ROBERT}

ABSTRACT. We give an elementary proof of the Fourier inversion formula (on the real line) based on the Poisson summation formula.

Let $f$ be a complex valued Schwartz function (i.e. all derivatives of $f$ decrease rapidly) on the real line. Its Fourier transform $\hat{f}$ is defined by

$$
\hat{f}(y)=\int_{-\infty}^{\infty} f(x) e^{-2 \pi i x y} d x
$$

(again in the Schwartz class), and we intend to prove the inversion $\hat{\hat{f}}(x)$ $=f(-x)$. Because $\left(\tau_{a} f\right)^{\wedge}(y)=e^{2 \pi i a y} \hat{f}(y), f(x+a)^{\hat{\imath}}=\hat{\hat{f}}(x-a)$ and it is enough to prove the inversion formula at the origin: $\hat{f}(0)=f(0)$. (Here, $\left(\tau_{a} f\right)(x)=f(x+a)$.)

1. The series $\sum_{m=-\infty}^{\infty} f(x+m)$ converges uniformly. Its sum is a continuous periodic function $F$ having Fourier coefficients

$$
\begin{aligned}
c_{n}(F) & =\int_{0}^{1} F(x) e^{-2 \pi i n x} d x=\sum_{n} \int_{0}^{1} f(x+m) e^{-2 \pi i n(x+m)} d x \\
& =\int_{-\infty}^{\infty} f(x) e^{-2 \pi i n x} d x=\hat{f}(n) \quad \text { (quickly decreasing) } .
\end{aligned}
$$

Hence the Fourier series $\sum \hat{f}(n) e^{2 \pi i n x}$ converges uniformly to $F$ and $x=0$ gives the Poisson summation formula $\Sigma_{n} f(n)=\Sigma_{m} \hat{f}(m)$.

2. We apply the Poisson formula to $f_{k}(x)=f(k x)(k \neq 0)$ and get

$$
\sum_{n} f(k n)=\sum_{m} \hat{f}(m / k) \cdot 1 /|k| .
$$

Letting $k \rightarrow \infty$, the left-hand side tends to $f(0)$ whereas the right-hand side is a Riemann sum approaching the integral $\int_{-\infty}^{\infty} \hat{f}(y) d y=\hat{\hat{f}}(0)$.

3. One can also use the Poisson formula

$$
\sum f(n)=\sum \hat{f}(n)=\sum \hat{\hat{f}}(n)
$$

Received by the editors January 12, 1976.

AMS (MOS) subject classifications (1970). Primary 42-00, 42A68.

- American Mathematical Society 1976 
with the new function $e^{2 \pi i a x} f$ getting

$$
\sum f(n) e^{2 \pi i a n}=\sum \hat{\hat{f}}(n) e^{-2 \pi i a n}=\sum \hat{\hat{f}}(-n) e^{2 \pi i a n}
$$

(for all real values of $a$ ). The identity of these continuous functions of $a$ implies the equality of their Fourier coefficients $f(n)=\hat{\hat{f}}(-n)$. This last method is essentially due to Gel'fand (cf. [1]).

The reader will observe that no hard theorem in integration is needed in these derivations, and the same pattern works as well over the $p$-adic fields $\mathbf{Q}_{p}$ instead of $\mathbf{R}$.

\section{REFERENCES}

1. N. J. Vilenkin, Special functions and the theory of group representations, "Nauka", Moscow, 1965; English transl., Transl. Math. Monographs, vol. 22, Chap. II, p. 3, Amer. Math. Soc., Providence, R.I., 1968. MR 35 \#420; 37 \#429.

Institut de Mathematiques, Chantemerle 20, CH-2000, Neuchatel, Switzerland 